\title{
Is there any "LSND anomaly"?
}

\section{A. Zhemchugov* (for the HARP-CDP group')}

Joint Institute for Nuclear Research

E-mail: zhemchugovejinr.ru

The LSND Collaboration reported a $3.8 \sigma$ excess of $\bar{v}_{\mathrm{e}}$ over background, in an experiment that dumped $800 \mathrm{MeV}$ protons into a water target. They interpreted this excess as evidence for $\bar{v}_{\mu} \rightarrow \bar{v}_{\mathrm{e}}$ oscillations, which led to the suggestion of 'sterile' neutrinos. LSND's claim was not confirmed by the MiniBooNE Collaboration, yet the origins of the LSND result were never clarified. The data from the HARP-CDP group on pion production by $800 \mathrm{MeV}$ protons are used in an independent calculation of LSND's $\bar{v}_{\mathrm{e}}$ background, also taking into account pion production by neutrons which had been ignored in LSND's calculations. We conclude that LSND's claim of a $3.8 \sigma$ excess cannot be upheld.

35th International Conference of High Energy Physics - ICHEP2010,

July 22-28, 2010

Paris France

* Speaker.

$\dagger$ The members of the HARP-CDP group are: A. Bolshakova, I. Boyko, G. Chelkov, D. Dedovich, A. Elagin, D. Emelyanov, M. Gostkin, A. Guskov, Z. Kroumchtein, Yu. Nefedov, K. Nikolaev and A. Zhemchugov from the Joint Institute for Nuclear Research, Dubna, Russian Federation; F. Dydak and J. Wotschack from CERN, Geneva, Switzerland; A. De Min from the Politecnico di Milano and INFN, Sezione di Milano-Bicocca, Milan, Italy; V. Ammosov, V. Gapienko, V. Koreshev, A. Semak, Yu. Sviridov, E. Usenko and V. Zaets from the Institute of High Energy Physics, Protvino, Russian Federation. 


\begin{tabular}{|l|llll|}
\hline & $\begin{array}{l}\text { LSND } \\
(\text { runs 1993-1995) }\end{array}$ & LSND “emulation” & $\begin{array}{l}\text { Geant4 } \\
\oplus \text { exp. data }\end{array}$ & $\begin{array}{l}\text { FLUKA } \\
\oplus \text { exp. data }\end{array}$ \\
\hline$\pi^{-} / \pi^{+}$ & - & 0.203 & 0.382 & 0.356 \\
$\bar{v}_{\mu}\left[v / P o T / \mathrm{cm}^{2}\right]$ & $0.8 \times 10^{-9}$ & $0.72 \times 10^{-9}$ & $0.76 \times 10^{-9}$ & $0.75 \times 10^{-9}$ \\
$\bar{v}_{\mathrm{e}}\left[\mathrm{v} / \mathrm{PoT} / \mathrm{cm}^{2}\right]$ & $0.65 \times 10^{-12}$ & $0.56 \times 10^{-12}$ & $0.96 \times 10^{-12}$ & $0.89 \times 10^{-12}$ \\
\hline
\end{tabular}

Table 1: Neutrino fluxes from muon decay at rest: HARP-CDP simulation results compared with the results published by LSND [1].

The LSND experiment reported an anomalous $3.8 \sigma$ excess of $\bar{v}_{e}$, interpreted as $\bar{v}_{\mu} \rightarrow \bar{v}_{\mathrm{e}}$ oscillation with $\Delta m^{2} \approx 1 \mathrm{eV}^{2}[1]$. This result has, until today, not been confirmed by other experiments. The HARP experiment measured pion production with a $1.5 \mathrm{GeV} / \mathrm{c}$ proton beam impinging on various target materials, including water and copper. These measurements were used to cross-check the calculation of LSND's $\bar{v}_{\mathrm{e}}$ background. Only data from the HARP large-angle spectrometer, analyzed by the HARP-CDP group, were used[2].

Two independent simulation programs have been developed. Hadron production has been simulated by Geant 4 [3] and FLUKA[ [4] codes. Then, pion production by protons has been adjusted to the HARP-CDP data, and pion production by neutrons has been tuned according to the results of other experiments[5]. In addition, we "emulated" the LSND procedure of neutrino flux calculation using the LSND's pion production parametrization[6], to demonstrate understanding of the LSND geometry. The results of our calculation are shown in Table $11^{1}$.

Comparing the LSND "emulation" and our best estimate based on Geant4 and FLUKA, adjusted to the experimental data, we conclude, that the larger part of the background of LSND's $\bar{v}_{\mathrm{e}}$ signal was underestimated by nearly a factor of 1.7. The causes were too small pion production cross-sections by protons and the neglect of pion production by neutrons, which, unlike protons, predominantly produce $\pi^{-}$rather than $\pi^{+}$. Thus, the claim of a $3.8 \sigma$ significance of the LSND anomaly cannot be upheld.

\section{References}

[1] A. Aguilar et al., Phys. Rev. D64 (2001) 112007

[2] V. Ammosov et al., Nucl. Instrum. Meth. A588 (2008) 294; V. Ammosov et al., Nucl. Instrum. Meth. A578 (2007) 119

[3] S. Agostinelli et al., Nucl. Instrum. Meth. A506 (2003) 250; J. Allison et al., IEEE Trans. Nucl. Sci. 53 (2006) 270

[4] G. Battistoni et al, AIP Conf. Proc. 896, 31-49, (2007); A. Fasso et al.,CERN-2005-10 (2005), INFN/TC-05/11, SLAC-R-773

[5] K.O. Oganesian, JETP 54 (1968) 1273; V.N. Baturin et al., JETP Lett., 30 (1979) 86

[6] We thank M. Sung for providing the LSND parametrization; the program code is available at http://hep.phys.lsu.edu/sung/lsnd/beammc/index.html

\footnotetext{
${ }^{1}$ The neutrino fluxes in the table are different from values shown at the conference. This discrepancy is explained by a computational mistake in our simulation, which has been found and eliminated after the conference. We note that this mistake did not affect the final conclusion.
} 